MATHEMATICS OF COMPUTATION

Volume 72, Number 244, Pages 1901-1916

S 0025-5718(03)01477-7

Article electronically published on January 8, 2003

\title{
MONIC INTEGER CHEBYSHEV PROBLEM
}

\author{
P. B. BORWEIN, C. G. PINNER, AND I. E. PRITSKER
}

Abstract. We study the problem of minimizing the supremum norm by monic polynomials with integer coefficients. Let $\mathcal{M}_{n}(\mathbb{Z})$ denote the monic polynomials of degree $n$ with integer coefficients. A monic integer Chebyshev polynomial $M_{n} \in \mathcal{M}_{n}(\mathbb{Z})$ satisfies

$$
\left\|M_{n}\right\|_{E}=\inf _{P_{n} \in \mathcal{M}_{n}(\mathbb{Z})}\left\|P_{n}\right\|_{E} .
$$

and the monic integer Chebyshev constant is then defined by

$$
t_{M}(E):=\lim _{n \rightarrow \infty}\left\|M_{n}\right\|_{E}^{1 / n} .
$$

This is the obvious analogue of the more usual integer Chebyshev constant that has been much studied.

We compute $t_{M}(E)$ for various sets, including all finite sets of rationals, and make the following conjecture, which we prove in many cases.

Conjecture. Suppose $\left[a_{2} / b_{2}, a_{1} / b_{1}\right]$ is an interval whose endpoints are consecutive Farey fractions. This is characterized by $a_{1} b_{2}-a_{2} b_{1}=1$. Then

$$
t_{M}\left[a_{2} / b_{2}, a_{1} / b_{1}\right]=\max \left(1 / b_{1}, 1 / b_{2}\right) .
$$

This should be contrasted with the nonmonic integer Chebyshev constant case, where the only intervals for which the constant is exactly computed are intervals of length 4 or greater.

\section{INTRODUCTION AND GENERAL RESULTS}

Define the uniform (sup) norm on a compact set $E \subset \mathbb{C}$ by

$$
\|f\|_{E}:=\sup _{z \in E}|f(z)| \text {. }
$$

We study the monic polynomials with integer coefficients that minimize the sup norm on the set $E$. Let $\mathcal{P}_{n}(\mathbb{C})$ and $\mathcal{P}_{n}(\mathbb{Z})$ be the classes of algebraic polynomials of degree at most $n$, respectively with complex and with integer coefficients. Similarly, we define the classes of monic polynomials $\mathcal{M}_{n}(\mathbb{C})$ and $\mathcal{M}_{n}(\mathbb{Z})$ of exact degree $n \in \mathbb{N}$. The problem of minimizing the uniform norm on $E$ by polynomials from $\mathcal{M}_{n}(\mathbb{C})$ is well known as the Chebyshev problem (see [1, 15], [17, 8], etc.). In the classical case, $E=[-1,1]$, the explicit solution of this problem is given by the

Received by the editor August 29, 2001 and, in revised form, December 20, 2001

2000 Mathematics Subject Classification. Primary 11C08; Secondary 30C10.

Key words and phrases. Chebyshev polynomials, integer Chebyshev constant, integer transfinite diameter.

Research of the authors was supported in part by the following grants: NSERC of Canada and MITACS (Borwein), NSF grant EPS-9874732 and matching support from the state of Kansas (Pinner), and NSF grant DMS-9996410 (Pritsker). 
monic Chebyshev polynomial of degree $n$ :

$$
T_{n}(x):=2^{1-n} \cos (n \arccos x), \quad n \in \mathbb{N} .
$$

Using a change of variable, we can immediately extend this to an arbitrary interval $[a, b] \subset \mathbb{R}$, so that

$$
t_{n}(x):=\left(\frac{b-a}{2}\right)^{n} T_{n}\left(\frac{2 x-a-b}{b-a}\right)
$$

is a monic polynomial with real coefficients and the smallest uniform norm on $[a, b]$ among all polynomials from $\mathcal{M}_{n}(\mathbb{C})$. In fact,

$$
\left\|t_{n}\right\|_{[a, b]}=2\left(\frac{b-a}{4}\right)^{n}, \quad n \in \mathbb{N}
$$

and we find that the Chebyshev constant for $[a, b]$ is given by

$$
t_{\mathbb{C}}([a, b]):=\lim _{n \rightarrow \infty}\left\|t_{n}\right\|_{[a, b]}^{1 / n}=\frac{b-a}{4} .
$$

The Chebyshev constant of an arbitrary compact set $E \subset \mathbb{C}$ is defined in a similar fashion:

$$
t_{\mathbb{C}}(E):=\lim _{n \rightarrow \infty}\left\|t_{n}\right\|_{E}^{1 / n},
$$

where $t_{n}$ is the Chebyshev polynomial of degree $n$ on $E$ (the monic polynomial of exact degree $n$ of minimal supremum norm on $E$ ). It is known that $t_{\mathbb{C}}(E)$ is equal to the transfinite diameter and the logarithmic capacity $\operatorname{cap}(E)$ of the set $E$ (cf. [17, pp. 71-75], [8] and [14 for the definitions and background material). by

An integer Chebyshev polynomial $Q_{n} \in \mathcal{P}_{n}(\mathbb{Z})$ for a compact set $E \subset \mathbb{C}$ is defined

$$
\left\|Q_{n}\right\|_{E}=\inf _{0 \neq P_{n} \in \mathcal{P}_{n}(\mathbb{Z})}\left\|P_{n}\right\|_{E},
$$

where the inf is taken over all polynomials from $\mathcal{P}_{n}(\mathbb{Z})$ which are not identically zero. Further, the integer Chebyshev constant (or integer transfinite diameter) for $E$ is given by

$$
t_{\mathbb{Z}}(E):=\lim _{n \rightarrow \infty}\left\|Q_{n}\right\|_{E}^{1 / n}
$$

The integer Chebyshev problem is also a classical subject of analysis and number theory (see [11, Ch. 10], [3], 2], 6], [7, [9, 16], [13] and the references therein). It does not require the polynomials to be monic. We define the associated quantities for the monic integer Chebyshev problem as follows. A monic integer Chebyshev polynomial $M_{n} \in \mathcal{M}_{n}(\mathbb{Z}), \operatorname{deg} M_{n}=n$, satisfies

$$
\left\|M_{n}\right\|_{E}=\inf _{P_{n} \in \mathcal{M}_{n}(\mathbb{Z})}\left\|P_{n}\right\|_{E} .
$$

The monic integer Chebyshev constant is then defined by

$$
t_{M}(E):=\lim _{n \rightarrow \infty}\left\|M_{n}\right\|_{E}^{1 / n}=\inf _{n}\left\|M_{n}\right\|_{E}^{1 / n},
$$

where the existence of this limit and the last equality follows by a standard argument presented in Lemma 3.1. The monic integer Chebyshev problem is quite different from the classical integer Chebyshev problem, as we show in this paper.

It is immediately clear from the definitions (1.4)-(1.7) that

$$
t_{M}(E) \geq t_{\mathbb{Z}}(E) \text {. }
$$


Note that, for any $P_{n} \in \mathcal{P}_{n}(\mathbb{Z})$,

$$
\left\|P_{n}\right\|_{E}=\left\|P_{n}\right\|_{E^{*}}
$$

where $E^{*}:=E \cup\{z: \bar{z} \in E\}$, because $P_{n}$ has real coefficients. Thus the (monic) integer Chebyshev problem on a compact set $E$ is equivalent to that on $E^{*}$, and we can assume that $E$ is symmetric with respect to the real axis ( $\mathbb{R}$-symmetric) without loss of generality.

Our first result shows that the monic integer Chebyshev constant coincides with the regular Chebyshev constant (capacity) for sufficiently large sets.

Theorem 1.1. If $E$ is $\mathbb{R}$-symmetric and $\operatorname{cap}(E) \geq 1$, then

$$
t_{M}(E)=\operatorname{cap}(E) .
$$

We remark that $t_{\mathbb{Z}}(E)=1$ for the sets $E$ with $\operatorname{cap}(E) \geq 1$. Indeed, $\left\|P_{n}\right\|_{E} \geq$ $(\operatorname{cap}(E))^{n}$ for any $P_{n} \in \mathcal{P}_{n}(\mathbb{Z})$ of exact degree $n$ (cf. [14, p. 155]). Thus $Q_{n}(z) \equiv 1$ is a minimizer for (1.4) in this case.

An argument going back to Kakeya (cf. 12] or [16]) gives

Proposition 1.2. Let $E \subset \mathbb{C}$ be a compact $\mathbb{R}$-symmetric set. If $\operatorname{cap}(E)<1$, then $t_{M}(E)<1$.

We show below that this statement cannot be significantly improved.

The monic integer Chebyshev constant shares a number of standard properties with $t_{\mathbb{Z}}(E)$ and $t_{\mathbb{C}}(E)$, such as the monotonicity property below.

Proposition 1.3. Let $E \subset F \subset \mathbb{C}$. Then

$$
t_{M}(E) \leq t_{M}(F) .
$$

Another generic property of importance is the following (see [5] and Theorem 2 of [8, Sect. VII.1]).

Proposition 1.4. Let $E \subset \mathbb{C}$ be a compact set. If $P_{n}^{-1}(E)$ is the inverse image of $E$ under $P_{n} \in \mathcal{M}_{n}(\mathbb{Z}), \operatorname{deg} P_{n}=n$, then

$$
t_{M}\left(P_{n}^{-1}(E)\right)=\left(t_{M}(E)\right)^{1 / n} .
$$

Perhaps, the most distinctive feature of $t_{M}(E)$ is that it may be different from zero even for a single point. For example (see section 2 below), suppose that $m, n \in \mathbb{Z}$, where $n \geq 2$ and $(m, n)=1$. Then

$$
t_{M}\left(\left\{\frac{m}{n}\right\}\right)=\frac{1}{n} .
$$

On the other hand, if $a \in \mathbb{R}$ is irrational, then

$$
t_{M}(\{a\})=0 .
$$

This result has several interesting consequences. Consider $E_{n}:=\left\{z: z^{n}=\right.$ $1 / 2\}, n \in \mathbb{N}$. It is obvious that $\operatorname{cap}\left(E_{n}\right)=t_{\mathbb{C}}\left(E_{n}\right)=0$ for any $n \in \mathbb{N}$. However, (1.11) and (1.10) imply that $t_{M}\left(E_{n}\right)=2^{-1 / n} \rightarrow 1$, as $n \rightarrow \infty$. Thus no uniform upper estimate of $t_{M}(E)$ in terms of $\operatorname{cap}(E)$ is possible, in contrast with the inequality $t_{\mathbb{Z}}(E) \leq \sqrt{\operatorname{cap}(E)}$ (see the results of Hilbert [10] and Fekete [4]).

We also note that $t_{M}(\{1 / \sqrt{2}\})=t_{M}(\{-1 / \sqrt{2}\})=0$ by (1.12), while $t_{M}(\{1 / \sqrt{2}\} \cup\{-1 / \sqrt{2}\})=1 / \sqrt{2}$ by (1.10) and (1.11). This shows that another 
well known property of capacity is not valid for $t_{M}(E)$. Namely, capacity (Chebyshev constant) for the union of two sets of zero capacity is still zero (cf. Theorem III.8 of [17, p. 57]).

Combining Proposition 1.3. Proposition 1.4 and (1.11), one can find the explicit values of the monic integer Chebyshev constant for many intervals and other sets.

Theorem 1.5. Let $n \in \mathbb{Z}$. The following relations hold true:

$$
\begin{aligned}
& t_{M}\left(\left[0, \frac{1}{n}\right]\right)=t_{M}\left(\left[\frac{n-1}{n}, 1\right]\right)=t_{M}\left(\left[-\frac{1}{n}, \frac{1}{n}\right]\right)=\frac{1}{n}, \quad n \geq 2, \\
& t_{M}\left(\left[-\frac{1}{\sqrt{n}}, \frac{1}{\sqrt{n}}\right]\right)=\sqrt{t_{M}\left(\left[0, \frac{1}{n}\right]\right)}=\frac{1}{\sqrt{n}}, \quad n \geq 2, \\
& t_{M}\left(\left[n, n+\frac{1}{2}\right]\right)=t_{M}\left(\left[n-\frac{1}{2}, n\right]\right)=t_{M}\left(\left[0, \frac{1}{2}\right]\right)=\frac{1}{2}, \\
& t_{M}([n, n+1])=t_{M}([0,1])=\sqrt{t_{M}\left(\left[0, \frac{1}{4}\right]\right)}=\frac{1}{2}
\end{aligned}
$$

and

$$
t_{M}([n, n+2])=t_{M}([-1,1])=\sqrt{t_{M}([0,1])}=\frac{1}{\sqrt{2}} .
$$

Also, if $E \subset[(1-\sqrt{2}) / 2,(1+\sqrt{2}) / 2]$ and $\{1 / 2\} \in E$, then

$$
t_{M}(E)=t_{M}\left(\left[\frac{1-\sqrt{2}}{2}, \frac{1+\sqrt{2}}{2}\right]\right)=t_{M}\left(\left\{\frac{1}{2}\right\}\right)=\frac{1}{2} .
$$

Of course, the above list of values can be extended further. It is worth mentioning that finding the value of $t_{\mathbb{Z}}([0,1])$ is a notoriously difficult problem, where we do not even have a current conjecture (see [3, [11, Ch. 10], [2] and [13]). From this point of view, the monic integer Chebyshev problem seems to be easier than its classical counterpart.

The rest of our paper is organized as follows. We consider the monic integer Chebyshev problem for finite sets in Section 2. Sections 3 and 4 contain proofs of the results from Sections 1 and 2 respectively. Section 5 is devoted to the study of Farey intervals, where we give some numerical results and state an interesting conjecture on the value of the monic integer Chebyshev constant.

\section{Finite SETS OF POINTS}

While finite numbers of integers can of course in no way affect $t_{M}(E)$, it is readily seen that the presence of noninteger rationals does restrict how small $t_{M}(E)$ can become, with

$$
\frac{a}{b} \in E, \quad b \geq 2 \Rightarrow t_{M}(E) \geq \frac{1}{b}
$$

(for a monic integer polynomial $P$ of degree $n$ we plainly have $\left|b^{n} P(a / b)\right| \geq 1$ ). Indeed for a finite set of rationals this bound is precise, as an immediate consequence of the following: 
Theorem 2.1. For any $k$ rational points

$$
\frac{a_{i}}{b_{i}}, \quad\left(a_{i}, b_{i}\right)=1, \quad i=1, \ldots, k,
$$

there is a monic integer polynomial $f(x)$ of degree $n$ for some positive integer $n$ with

$$
f\left(\frac{a_{i}}{b_{i}}\right)=\frac{1}{b_{i}^{n}}, \quad i=1, \ldots, k .
$$

Corollary 2.2. If $E=\left\{\frac{a_{1}}{b_{1}}, \ldots, \frac{a_{k}}{b_{k}}\right\}$ with the $a_{i} / b_{i}$ rationals written in their lowest terms and $b_{i} \geq 2$, then

$$
t_{M}(E)=\max _{i=1, \ldots, k} \frac{1}{b_{i}} .
$$

Two consecutive Farey fractions: It is perhaps worth noting that in the case of two consecutive Farey fractions

$$
\frac{a_{2}}{b_{2}}<\frac{a_{1}}{b_{1}}, \quad a_{1} b_{2}-a_{2} b_{1}=1,
$$

it is easy to explicitly write down a polynomial satisfying Theorem 2.1 (or any congruence feasible values):

If $n \geq 2$ with

$$
a_{i}^{n} \equiv A_{i} \quad \bmod b_{i}, \quad i=1,2,
$$

then

$$
f(x)=x^{n}+\left(\frac{A_{1}-a_{1}^{n}}{b_{1}}\right)\left(b_{2} x-a_{2}\right)^{n-1}+\left(\frac{A_{2}-a_{2}^{n}}{b_{2}}\right)\left(a_{1}-b_{1} x\right)^{n-1}
$$

has $f\left(a_{i} / b_{i}\right)=A_{i} / b_{i}^{n}, i=1,2$.

Moreover, if $a_{3} / b_{3}$ is the next Farey fraction between them, $a_{3}=a_{1}+a_{2}, b_{3}=$ $b_{1}+b_{2}$, and $n \geq 3$ with $a_{i}^{n} \equiv A_{i} \bmod b_{i}, i=1,2,3$, then the polynomial

$$
\tilde{f}(x)=f(x)+\left(\frac{A_{3}-a_{3}^{n}}{b_{3}}-\frac{A_{2}-a_{2}^{n}}{b_{2}}-\frac{A_{1}-a_{1}^{n}}{b_{1}}\right)\left(b_{2} x-a_{2}\right)^{j}\left(a_{1}-b_{1} x\right)^{n-1-j}
$$

satisfies $\tilde{f}\left(a_{i} / b_{i}\right)=A_{i} / b_{i}^{n}, i=1,2,3$, for any $1 \leq j \leq n-2$.

For higher degree algebraic numbers which are not algebraic integers (adding an algebraic integer can plainly not change the monic integer Chebyshev constant), the presence of a full set of conjugates similarly leads to a lower bound. In particular, if $E$ contains all the roots $\alpha_{1}, \ldots, \alpha_{d}$ of an irreducible integer polynomial of degree $d$ and lead coefficient $b \geq 2$, then

$$
t_{M}(E) \geq \frac{1}{b^{\frac{1}{d}}}
$$

(since for any monic integer polynomial $P$ of degree $n$ the quantity $b^{n} \prod_{i=1}^{d} P\left(\alpha_{i}\right)$ is an integer and necessarily nonzero). Proposition 1.4 and Corollary 2.2 can be used to furnish nonrational cases where such a bound is sharp. However, if $E$ consists of a set of conjugates missing at least one real or pair of complex conjugates, then in fact $t_{M}(E)=0$. Similarly if $E$ consists of a finite number of transcendentals. These (and other similar examples) follow at once from the following result: 
Theorem 2.3. Suppose that $S=\left\{\alpha_{1}, \ldots, \alpha_{k}\right\}$ is a set of $k$ numbers, with the $\alpha_{i}$ transcendental or algebraic of degree more than $k$. If $S$ is closed under complex conjugation, then for any $\varepsilon \in(0,1)$ there is a monic integer polynomial $F$ of degree $n=n\left(\alpha_{1}, \ldots, \alpha_{k}\right)$ with $\left|F\left(\alpha_{i}\right)\right|<\varepsilon, i=1, \ldots, k$.

\section{Proofs For Section 1}

Lemma 3.1. The limit defining $t_{M}(E)$ in (1.7) by

$$
t_{M}(E):=\lim _{n \rightarrow \infty}\left\|M_{n}\right\|_{E}^{1 / n}
$$

exists. Furthermore,

$$
\lim _{n \rightarrow \infty}\left\|M_{n}\right\|_{E}^{1 / n}=\inf _{n}\left\|M_{n}\right\|_{E}^{1 / n} .
$$

Proof. The argument is identical to the classical Chebyshev constant case. Indeed, let

$$
v_{n}:=\left\|M_{n}\right\|_{E}=\inf _{P_{n} \in \mathcal{M}_{n}(\mathbb{Z})}\left\|P_{n}\right\|_{E}, \quad n \in \mathbb{N}
$$

Then

$$
v_{k+m} \leq\left\|M_{k} M_{m}\right\|_{E} \leq\left\|M_{k}\right\|_{E}\left\|M_{m}\right\|_{E}=v_{k} v_{m} .
$$

On setting $a_{n}=\log v_{n}$, we obtain that

$$
a_{k+m} \leq a_{k}+a_{m}, \quad k, m \in \mathbb{N} .
$$

Hence

$$
\lim _{n \rightarrow \infty} \frac{a_{n}}{n}=\lim _{n \rightarrow \infty} \log \left(v_{n}\right)^{1 / n}
$$

exists (possibly as $-\infty$ ) by the lemma on page 73 of 17 .

If $\inf _{n}\left\|M_{n}\right\|_{E}^{1 / n}=\liminf _{n \rightarrow \infty}\left\|M_{n}\right\|_{E}^{1 / n}$, then the second statement of this lemma follows from the above. Otherwise, we have

$$
\inf _{n}\left\|M_{n}\right\|_{E}^{1 / n}=\left\|M_{k}\right\|_{E}^{1 / k}
$$

for a particular $k \in \mathbb{N}$. But then the sequence of polynomials $\left\{\left(M_{k}\right)^{m}\right\}_{m=0}^{\infty}$ satisfies

$$
\left\|M_{k}\right\|_{E}^{1 / k}=\lim _{m \rightarrow \infty}\left\|\left(M_{k}\right)^{m}\right\|_{E}^{1 /(k m)} \geq t_{M}(E) \geq \inf _{n}\left\|M_{n}\right\|_{E}^{1 / n}=\left\|M_{k}\right\|_{E}^{1 / k} .
$$

Proof of Theorem 1.1. Let $T_{n}(z)=z^{n}+a_{n-1}^{(n)} z^{n-1}+a_{n-2}^{(n)} z^{n-2}+\ldots+a_{0}^{(n)}, n \in \mathbb{N}$, be the Chebyshev polynomials for $E$. Since $E$ is $\mathbb{R}$-symmetric, the coefficients of Chebyshev polynomials are real (cf. [17, p. 72]). By the definition of (1.3), for any $\varepsilon>0$ there exists $N \in \mathbb{N}$ such that

$$
\left\|T_{n}\right\|_{E}^{1 / n} \leq \operatorname{cap}(E)+\varepsilon, \quad n \geq N .
$$

We shall construct a sequence of monic polynomials with integer coefficients and small norms from the Chebyshev polynomials on $E$. This is done by the following inductive procedure. Consider $n \geq N$ and the polynomial $T_{n}-\left(a_{n-1}^{(n)}-\left[a_{n-1}^{(n)}\right]\right) T_{n-1}$, with the two highest coefficients integers. We have that

$$
\left\|T_{n}-\left(a_{n-1}^{(n)}-\left[a_{n-1}^{(n)}\right]\right) T_{n-1}\right\|_{E} \leq(\operatorname{cap}(E)+\varepsilon)^{n}+(\operatorname{cap}(E)+\varepsilon)^{n-1} .
$$


Continuing in the same fashion, we eliminate the fractional parts of all coefficients from the $n$-th to $(N+1)$-st, and obtain the following estimate:

$$
\left\|z^{n}+\left[a_{n-1}^{(n)}\right] z^{n-1}+\ldots+[\cdot] z^{N+1}+\sum_{k=0}^{N} b_{k} z^{k}\right\|_{E} \leq \sum_{l=N+1}^{n}(\operatorname{cap}(E)+\varepsilon)^{l} .
$$

Note that

$$
\left\|\sum_{k=0}^{N}\left(b_{k}-\left[b_{k}\right]\right) z^{k}\right\|_{E} \leq \sum_{k=0}^{N}\left\|z^{k}\right\|_{E}=: A(N),
$$

where $A(N)>0$ depends only on $N$ and the set $E$. Hence we have from (3.1) that

$$
\begin{aligned}
\| z^{n}+\left[a_{n-1}^{(n)}\right] z^{n-1}+\ldots+[\cdot] & z^{N+1}+\sum_{k=0}^{N}\left[b_{k}\right] z^{k} \|_{E} \\
& \leq(\operatorname{cap}(E)+\varepsilon)^{N+1} \frac{(\operatorname{cap}(E)+\varepsilon)^{n-N}-1}{\operatorname{cap}(E)+\varepsilon-1}+A(N),
\end{aligned}
$$

because $\operatorname{cap}(E) \geq 1$. Denote the constructed polynomial by $Q_{n} \in \mathcal{M}(\mathbb{Z}), n \in \mathbb{N}$. It follows that

$$
\limsup _{n \rightarrow \infty}\left\|Q_{n}\right\|_{E}^{1 / n} \leq \operatorname{cap}(E)+\varepsilon
$$

and

$$
t_{M}(E) \leq \operatorname{cap}(E)+\varepsilon .
$$

Letting $\varepsilon \rightarrow 0$ and recalling that $t_{M}(E) \geq t_{\mathbb{C}}(E)=\operatorname{cap}(E)$ by definition, we finish the proof.

Proof of Proposition 1.2. See Kakeya's proof in [12] or [16].

Proof of Proposition 1.3. This proposition readily follows from the inequality

$$
\left\|p_{n}\right\|_{E} \leq\left\|p_{n}\right\|_{F}
$$

valid for any polynomial $p_{n}(z)$.

Proof of Proposition 1.4. The following argument is due to Fekete [5. Let $M_{k}(z)$, $k \in \mathbb{N}$, be monic integer Chebyshev polynomials for $E$, and let $M_{k}^{*}(z), k \in \mathbb{N}$, be monic integer Chebyshev polynomials for $E^{*}:=P_{n}^{-1}(E)$. It follows from the definition that

$$
\left\|M_{k n}^{*}\right\|_{E^{*}} \leq\left\|M_{k} \circ P_{n}\right\|_{E^{*}} \leq\left\|M_{k}\right\|_{E}, \quad k \in \mathbb{N} .
$$

Hence

$$
t_{M}\left(E^{*}\right) \leq\left(t_{M}(E)\right)^{1 / n} .
$$

To prove the opposite inequality, we consider the roots $z_{i}, i=1, \ldots, n$, of the equation $P_{n}(z)-w=0$, where $w \in E$ is fixed. If $z_{j}^{*}, j=1, \ldots, k$, are the roots of $M_{k}^{*}(z)$, then we have that

$$
\left|\prod_{i=1}^{n} M_{k}^{*}\left(z_{i}\right)\right|=\left|\prod_{i=1}^{n} \prod_{j=1}^{k}\left(z_{i}-z_{j}^{*}\right)\right|=\left|\prod_{j=1}^{k} \prod_{i=1}^{n}\left(z_{j}^{*}-z_{i}\right)\right|=\left|\prod_{j=1}^{k}\left(P_{n}\left(z_{j}^{*}\right)-w\right)\right| .
$$


Note that $Q_{k}(w):=\prod_{j=1}^{k}\left(w-P_{n}\left(z_{j}^{*}\right)\right)$ is a monic polynomial in $w$, with integer coefficients. Indeed, its coefficients are symmetric functions in the $z_{j}^{*}$ 's, which are integers by the fundamental theorem on symmetric forms. Thus we obtain that

$$
\left\|M_{k}\right\|_{E} \leq\left\|Q_{k}\right\|_{E} \leq\left(\left\|M_{k}^{*}\right\|_{E^{*}}\right)^{n}, \quad k \in \mathbb{N}
$$

and

$$
t_{M}(E) \leq\left(t_{M}\left(E^{*}\right)\right)^{n}
$$

Proof of Theorem 1.5. We first prove (1.13). The sequence of polynomials $\left\{z^{k}\right\}_{k=0}^{\infty}$ shows that $t_{M}([0,1 / n]) \leq 1 / n$ and $t_{M}([-1 / n, 1 / n]) \leq 1 / n$. On the other hand, we have that $t_{M}([0,1 / n]) \geq t_{M}(\{1 / n\})=1 / n$ by Proposition [1.3 and (1.11). The remaining equality for $t_{M}([1-1 / n, 1])$ follows from Proposition 1.4 , by using the change of variable $z \rightarrow 1-z$.

Applying the substitution $z \rightarrow z^{2}$, we obtain (1.14) from Proposition 1.4 and (1.13).

Note that $t_{M}([0,1 / 2])=1 / 2$ follows from (1.13). We can now map $[0,1 / 2]$ to $[n, n+1 / 2]$ by $z \rightarrow z+n$ (or to $[n-1 / 2, n]$ by $z \rightarrow n-z$ ), and apply Proposition 1.4 to prove (1.15). Similarly, (1.16) is obtained from Proposition 1.4 and (1.13) by the transformations $z \rightarrow z-n$ mapping $[n, n+1] \rightarrow[0,1]$, and $z \rightarrow z(1-z)$ mapping $[0,1] \rightarrow[0,1 / 4]$. The same argument applies to (1.17), where we first map $[n, n+2] \rightarrow[-1,1]$ with $z \rightarrow z-n-1$ and then map $[-1,1] \rightarrow[0,1]$ with $z \rightarrow z^{2}$.

Observe that

$$
|z(1-z)| \leq 1 / 4, \quad z \in\left[\frac{1-\sqrt{2}}{2}, \frac{1+\sqrt{2}}{2}\right]
$$

Hence

$$
t_{M}\left(\left[\frac{1-\sqrt{2}}{2}, \frac{1+\sqrt{2}}{2}\right]\right) \leq \frac{1}{2} .
$$

For $1 / 2 \in E \subset[(1-\sqrt{2}) / 2,(1+\sqrt{2}) / 2]$, Proposition 1.3 and (1.11) give that

$$
\frac{1}{2}=t_{M}\left(\left\{\frac{1}{2}\right\}\right) \leq t_{M}(E) \leq t_{M}\left(\left[\frac{1-\sqrt{2}}{2}, \frac{1+\sqrt{2}}{2}\right]\right) \leq \frac{1}{2} .
$$

It is clear that the segment $\left[\frac{1-\sqrt{2}}{2}, \frac{1+\sqrt{2}}{2}\right]$ can be replaced here by the lemniscate $\{z \in \mathbb{C}:|z(1-z)| \leq 1 / 4\}$.

\section{Proofs for Section 2}

Proof of Theorem 2.1. Set

$$
\begin{aligned}
E(j) & :=\prod_{i<j}\left(a_{j} b_{i}-a_{i} b_{j}\right), \quad j=2, \ldots, k, \\
D & :=\operatorname{lcm}[E(2), \ldots, E(k)],
\end{aligned}
$$


and write $D:=D_{1}(j) D_{2}(j), E(j):=E_{1}(j) E_{2}(j)$, where

$$
\begin{array}{ll}
D_{1}(j)=\prod_{\substack{p^{\alpha} \| D \\
p \mid b_{j}}} p^{\alpha}, & D_{2}(j)=\prod_{\substack{p^{\alpha} \| \mid D \\
p \nmid b_{j}}} p^{\alpha}, \\
E_{1}(j)=\prod_{\substack{\alpha \\
p^{\alpha} \| E(j) \\
p \mid b_{j}}} p^{\alpha}, & E_{2}(j)=\prod_{\substack{p^{\alpha} \| \mid E(j) \\
p \nmid b_{j}}} p^{\alpha} .
\end{array}
$$

Take $m$ to be a positive integer large enough that

$$
p^{\alpha} \mid D \Rightarrow \alpha<m
$$

and choose $n \geq k m$ such that for $j=1, \ldots, k$

$$
\begin{aligned}
a_{j}^{n} & \equiv 1 \bmod b_{j}^{k m}, \\
b_{j}^{n} & \equiv 1 \bmod D_{2}(j)^{k m} .
\end{aligned}
$$

Choose integers $l_{i}$ such that

$$
a_{i} l_{i} \equiv 1 \bmod b_{i},
$$

and write $a_{i} l_{i}-b_{i} f_{i}=1$.

The proof proceeds by induction on the number of rationals $1 \leq r \leq k$, constructing a polynomial

$$
F_{r}(x)=x^{n}+\sum_{i=0}^{n-(k+1-r) m} \beta_{i, r} x^{i}
$$

with $F_{r}\left(a_{j} / b_{j}\right)=1 / b_{j}^{n}, j=1, \ldots, r$.

The first step, $r=1$, is easy;

$$
F_{1}(x):=x^{n}+\left(\frac{1-a_{1}^{n}}{b_{1}^{k m}}\right)\left(l_{1} x-f_{1}\right)^{n-k m} .
$$

Next, given $F_{r}(x)$ with $r<k$, we construct $F_{r+1}(x)$. This amounts to finding an integer polynomial $Q(x)$, of degree at most $n-(k-r) m-r$, such that

$$
F_{r+1}(x)=F_{r}(x)+Q(x) \prod_{i=1}^{r}\left(b_{i} x-a_{i}\right)
$$

has $F_{r+1}\left(a_{r+1} / b_{r+1}\right)=1 / b_{r+1}^{n}$. Thus it is enough if

$$
\frac{1}{b_{r+1}^{n}}=F_{r}\left(\frac{a_{r+1}}{b_{r+1}}\right)+\frac{E(r+1)}{b_{r+1}^{r}} \frac{A}{b_{r+1}^{n-(k-r) m-r}},
$$

for some integer $A$, since we can then take

$$
Q(x)=A\left(l_{r+1} x-f_{r+1}\right)^{n-(k-r) m-r} .
$$

For this we require that $b_{r+1}^{(k-r) m} E(r+1)$ divides

$$
B:=b_{r+1}^{n} F_{r}\left(\frac{a_{r+1}}{b_{r+1}}\right)-1=\left(a_{r+1}^{n}-1\right)+\sum_{j=0}^{n-(k+1-r) m} \beta_{j, r} a_{r+1}^{j} b_{r+1}^{n-j} .
$$


Clearly from $E_{1}(r+1) \mid D_{1}(r+1)$ and the definition of $m$ we have $E_{1}(r+1) \mid b_{r+1}^{m}$, and $b_{r+1}^{(k-r) m} E_{1}(r+1) \mid b_{r+1}^{(k+1-r) m}$. So from (4.1) and $r \geq 1$ we certainly have that $b_{r+1}^{(k-r) m} E_{1}(r+1)$ divides $\left(a_{r+1}^{n}-1\right)$, and $b_{r+1}^{n-j}$ for $j \leq n-(k+1-r) m$, and hence $B$. Thus it remains to check that $E_{2}(r+1)$ divides $B$.

Suppose that $p^{\alpha}|| E_{2}(r+1)$. Then $p \mid\left(b_{j} a_{r+1}-a_{j} b_{r+1}\right)$ for some nonempty subset, $S$ say, of the $1 \leq j \leq r$. Note that since $p \nmid b_{r+1}$ we have $p \nmid b_{j}$ and $b_{j}^{n} \equiv 1 \bmod p^{m k}$ for all $j \in S \cup\{r+1\}$ from (4.2). Hence, choosing integers $\bar{b}_{j}$ with $b_{j} \bar{b}_{j} \equiv 1 \bmod p^{m k}$ for $j \in S \cup\{r+1\}$, we have

$$
0=b_{j}^{n} F_{r}\left(a_{j} / b_{j}\right)-1 \equiv F_{r}\left(a_{j} \bar{b}_{j}\right)-1 \bmod p^{m k},
$$

for the $j \in S$, with $B \equiv F_{r}\left(a_{r+1} \bar{b}_{r+1}\right)-1 \bmod p^{m k}$ and $p^{\alpha} \| \prod_{j \in S}\left(a_{r+1} \bar{b}_{r+1}-a_{j} \bar{b}_{j}\right)$. Thus we can successively divide $F_{r}(x)-1$ by $\left(x-a_{j} \bar{b}_{j}\right)$ for the $j \in S$ (assume we proceeed in order of increasing $j$ ). In particular, after dealing with a subset $S^{\prime}$ of the $j$ in $S$ we can write

$$
F_{r}(x)-1 \equiv T_{S^{\prime}}(x) \prod_{j \in S^{\prime}}\left(x-a_{j} \bar{b}_{j}\right) \quad \bmod p^{\left(k+1-\left|S^{\prime}\right|\right) m}
$$

for some integer polynomial $T_{S^{\prime}}(x)$, where $p^{m} \nmid \prod_{j \in S^{\prime}}\left(a_{i} \bar{b}_{i}-a_{j} \bar{b}_{j}\right)$ (as $p^{m} \nmid E(i)$ ) and $F_{r}\left(a_{i} \bar{b}_{i}\right)-1 \equiv 0 \bmod p^{k m}$ imply that

$$
T_{S^{\prime}}\left(a_{i} \bar{b}_{i}\right) \equiv 0 \quad \bmod p^{\left(k+1-\left|S^{\prime}\right|-1\right) m}
$$

for any remaining $i \in S \backslash S^{\prime}$. So

$$
\begin{aligned}
B & \equiv T_{S}\left(a_{r+1} \bar{b}_{r+1}\right) \prod_{j \in S}\left(a_{r+1} \bar{b}_{r+1}-a_{j} \bar{b}_{j}\right) \bmod p^{(k+1-|S|) m} \\
& \equiv 0 \quad \bmod p^{\alpha},
\end{aligned}
$$

as claimed.

Proof of Theorem 2.3. Suppose that we have a set of $k$ numbers as in the statement of Theorem 2.3 .

We first show that for any $1>\varepsilon>0$ there is a nonzero integer polynomial $P(x)=x^{j} Q(x)$ with $j \leq\left(\begin{array}{c}k \\ 2\end{array}\right)$ and $Q$ of degree at most $k$, with $0<\left|P\left(\alpha_{i}\right)\right|<\varepsilon / k$, $i=1, \ldots, k$, and $P\left(\alpha_{i}\right) \neq P\left(\alpha_{l}\right)$ when $\alpha_{i} / \alpha_{l}$ is not a root of unity. This essentially follows from Minkowski's theorem on linear forms: Taking an arbitrary real $\alpha_{k+1} \neq$ $\alpha_{i}, i=1, \ldots, k$, we can find a nonzero $\left(a_{0}, \ldots, a_{k}\right) \in \mathbb{Z}^{k+1}$ with

$$
\left|a_{0}+a_{1} \alpha_{i}+\cdots+a_{k} \alpha_{i}^{k}\right| \leq \frac{\varepsilon}{k \max \left\{1,\left|\alpha_{i}\right|\right\}^{\frac{1}{2} k(k-1)}},
$$

if $\alpha_{i}, i=1, \ldots, k$, is real, and for any pairs of complex conjugate $\alpha_{i}$

$$
\begin{aligned}
& \left|a_{0}+a_{1} \Re \alpha_{i}+\cdots+a_{k} \Re \alpha_{i}^{k}\right| \leq \frac{\varepsilon}{\sqrt{2} k \max \left\{1,\left|\alpha_{i}\right|\right\}^{\frac{1}{2} k(k-1)}}, \\
& \left|a_{0}+a_{1} \Im \alpha_{i}+\cdots+a_{k} \Im \alpha_{i}^{k}\right| \leq \frac{\varepsilon}{\sqrt{2} k \max \left\{1,\left|\alpha_{i}\right|\right\}^{\frac{1}{2} k(k-1)}},
\end{aligned}
$$

and

$$
\left|a_{0}+a_{1} \alpha_{k+1}+\cdots+a_{k} \alpha_{k+1}^{k}\right| \leq \frac{D k^{k} \prod_{i=1}^{k} \max \left\{1,\left|\alpha_{i}\right|\right\}^{\frac{1}{2} k(k-1)}}{\varepsilon^{k}},
$$


where

$$
D=\left|\operatorname{det}\left(\begin{array}{ccc}
1 & \cdots & \alpha_{1}^{k} \\
\vdots & & \vdots \\
1 & \cdots & \alpha_{k+1}^{k}
\end{array}\right)\right|=\prod_{i<j}\left|\alpha_{i}-\alpha_{j}\right| \neq 0
$$

Taking $Q(x)=a_{0}+\cdots+a_{k} x^{k}$, we plainly have $Q\left(\alpha_{i}\right) \neq 0\left(\right.$ since $\left.\left[\mathbb{Q}\left(\alpha_{i}\right): \mathbb{Q}\right]>k\right)$ and $\alpha_{i}^{j} Q\left(\alpha_{i}\right) \neq \alpha_{l}^{j} Q\left(\alpha_{l}\right)$, when $\alpha_{i} / \alpha_{l}$ is not a root of unity, for at least one $0 \leq$ $j \leq k(k-1) / 2\left(\right.$ since when $\alpha_{i} / \alpha_{l}$ is not a root of unity $Q\left(\alpha_{l}\right) / Q\left(\alpha_{i}\right)=\left(\alpha_{i} / \alpha_{l}\right)^{j}$ for at most one integer $j$, and there are at most $k(k-1) / 2$ such pairings with $i<l)$. Choosing $P(x)=x^{j} Q(x)$ for such a $j$ then has the desired property.

To complete the proof of Theorem 2.3. take the polynomial $P(x)$ as above, and an $n>k^{2}(k+1) / 2$ such that $\alpha_{i}^{n}=\alpha_{l}^{n}$ whenever $\alpha_{i} / \alpha_{l}$ is a root of unity, and solve the linear system

$$
A_{1} P\left(\alpha_{i}\right)+A_{2} P\left(\alpha_{i}\right)^{2}+\cdots+A_{m} P\left(\alpha_{i}\right)^{m}=-\alpha_{i}^{n}, \quad i=1, \ldots, m,
$$

where $P\left(\alpha_{1}\right), \ldots, P\left(\alpha_{m}\right)$ are the distinct values of $P\left(\alpha_{i}\right)$ (any remaining $\alpha_{l}$ with $P\left(\alpha_{l}\right)=P\left(\alpha_{i}\right), \alpha_{l}^{n}=\alpha_{i}^{n}$, will merely repeat one of these equations). This will have a solution, since

$$
\left|\operatorname{det}\left(\begin{array}{ccc}
P\left(\alpha_{1}\right) & \cdots & P\left(\alpha_{1}\right)^{m} \\
\vdots & & \vdots \\
P\left(\alpha_{m}\right) & \cdots & P\left(\alpha_{m}\right)^{m}
\end{array}\right)\right|=\left|P\left(\alpha_{1}\right)\right| \cdots\left|P\left(\alpha_{m}\right)\right| \prod_{i<j}\left|P\left(\alpha_{i}\right)-P\left(\alpha_{j}\right)\right| \neq 0 .
$$

Moreover, since the complex $\alpha_{i}$ come in complex conjugate pairs, the solution $A_{1}, \ldots, A_{m}$ will be real. Hence taking $b_{j}=\left[A_{j}\right], j=1, \ldots, m$, gives a monic integer polynomial

$$
F(x)=x^{n}+b_{m} P(x)^{m}+b_{m-1} P(x)^{m-1}+\cdots+b_{1} P(x)
$$

with

$$
\left|F\left(\alpha_{i}\right)\right|=\left|\sum_{j=1}^{m}\left\{A_{j}\right\} P\left(\alpha_{i}\right)^{j}\right| \leq \sum_{j=1}^{m}(\varepsilon / k)^{j}<\varepsilon .
$$

\section{Intervals OF CONSECUtive FAREy numbers}

Conjecture 5.1. Suppose $\left[a_{2} / b_{2}, a_{1} / b_{1}\right]$ is an interval whose endpoints are consecutive Farey fractions. This is characterized by $a_{1} b_{2}-a_{2} b_{1}=1$. Then

$$
t_{M}\left[a_{2} / b_{2}, a_{1} / b_{1}\right]=\max \left(1 / b_{1}, 1 / b_{2}\right) .
$$

From Corollary 2.2 we have

$$
t_{M}\left[a_{2} / b_{2}, a_{1} / b_{1}\right] \geq \max \left(1 / b_{1}, 1 / b_{2}\right),
$$

and by Theorem 1.5 the conjecture holds on intervals of the form $[0,1 / n]$. The following table gives enough solutions to fill in all Farey intervals with denominator less than 22. On the remaining intervals $x$ works or the symmetry $x \rightarrow m \pm x$ works. 
The computations for the table are done with LLL. As in section 2, for certain $n$, we can find a polynomial $p$ of degree $n$ that satisfies $p\left(a_{2} / b_{2}\right)=1 / b_{2}^{n}$ and $p\left(a_{1} / b_{1}\right)=1 / b_{1}^{n}$. One now constructs a basis

$$
\begin{aligned}
B:=\left(p(x),\left(b_{1} x-a_{1}\right)\left(b_{2} x-a_{2}\right), x\left(b_{1} x-a_{1}\right)\left(b_{2} x-a_{2}\right),\right. \\
\left.\ldots, x^{n-3}\left(b_{1} x-a_{1}\right)\left(b_{2} x-a_{2}\right)\right)
\end{aligned}
$$

and we reduce the basis with respect to the norm

$$
\left(\int_{a_{2} / b_{2}}^{a_{1} / b_{1}} p(x)^{2} d x\right)^{1 / 2} .
$$

We then search the reduced basis for solutions of the conjecture. These calculations were done in Maple using an LLL implementation that can accommodate reduction with respect to any positive definite quadratic form. (This was implemented by Kevin Hare, and we would like to thank him for his code.)

Here $T\left(a_{2} / b_{2}, a_{1} / b_{1}\right)$ is a polynomial that satisfies

$$
\left\|T\left(a_{2} / b_{2}, a_{1} / b_{1}\right)\right\|_{\left[a_{2} / b_{2}, a_{1} / b_{1}\right]}=\max \left(1 / b_{1}, 1 / b_{2}\right)^{\operatorname{deg} T},
$$

so that Conjecture 5.1 holds on $\left[a_{2} / b_{2}, a_{1} / b_{1}\right]$ by Lemma 3.1] There is no guarantee that it is the lowest degree example.

$$
\begin{aligned}
T(1 / 3,2 / 5)= & x^{2}-3 x+1 \\
T(1 / 4,2 / 7)= & x^{2}-4 x+1 \\
T(2 / 5,3 / 7)= & x^{4}-716 x^{3}+890 x^{2}-369 x+51 \\
T(1 / 3,3 / 8)= & x^{2}-3 x+1 \\
T(3 / 8,2 / 5)= & x^{2}-3 x+1 \\
T(1 / 5,2 / 9)= & -x^{3}-20 x^{2}+9 x-1 \\
T(3 / 7,4 / 9)= & -x^{3}+37 x^{2}-32 x+7 \\
T(2 / 7,3 / 10)= & -x^{6}+1151931 x^{5}-1691236 x^{4}+993150 x^{3}-291587 x^{2} \\
& +42802 x-2513 \\
T(1 / 6,2 / 11)= & -x^{3}-30 x^{2}+11 x-1 \\
T(1 / 4,3 / 11)= & x^{2}-4 x+1 \\
T(3 / 11,2 / 7)= & -x^{6}-2359829 x^{5}+3291253 x^{4}-1836029 x^{3}+512089 x^{2} \\
& -71410 x+3983 \\
T(1 / 3,4 / 11)= & x^{2}-6 x+2 \\
T(4 / 11,3 / 8)= & -x^{4}+830 x^{3}-928 x^{2}+346 x-43 \\
T(4 / 9,5 / 11)= & -x^{9}-29635158678 x^{8}+106792009997 x^{7}-168361710540 x^{6} \\
& +151671807240 x^{5}-85396766648 x^{4}+30771806151 x^{3} \\
& -6930101424 x^{2}+891832252 x-50211113 \\
T(2 / 5,5 / 12)= & x^{2}+2 x-1 \\
T(5 / 12,3 / 7)= & -x^{3}-26 x^{2}+23 x-5 \\
T(1 / 7,2 / 13)= & -x^{3}-42 x^{2}+13 x-1 \\
&
\end{aligned}
$$




$$
\begin{aligned}
& T(2 / 9,3 / 13)=-x^{3}-20 x^{2}+9 x-1 \\
& T(3 / 10,4 / 13)=-x^{6}-2627119 x^{5}+3994979 x^{4}-2429980 x^{3}+739017 x^{2} \\
& -112375 x+6835 \\
& T(3 / 8,5 / 13)=x^{2}-3 x+1 \\
& T(5 / 13,2 / 5)=x^{2}-3 x+1 \\
& T(5 / 11,6 / 13)=x^{5}+131482 x^{4}-240886 x^{3}+165494 x^{2}-50532 x+5786 \\
& T(1 / 5,3 / 14)=x^{2}-5 x+1 \\
& T(3 / 14,2 / 9)=-x^{3}+106 x^{2}-46 x+5 \\
& T(1 / 3,5 / 14)=x^{2}-6 x+2 \\
& T(5 / 14,4 / 11)=x^{5}+98683 x^{4}-142309 x^{3}+76957 x^{2}-18496 x+1667 \\
& T(1 / 8,2 / 15)=-x^{4}+4112 x^{3}-1602 x^{2}+208 x-9 \\
& T(1 / 4,4 / 15)=x^{2}-8 x+2 \\
& T(4 / 15,3 / 11)=x^{5}+162382 x^{4}-175226 x^{3}+70906 x^{2}-12752 x+860 \\
& T(6 / 13,7 / 15)=-x^{12}+72422527702901325 x^{11}-369852358365610457 x^{10} \\
& +858538529519890462 x^{9}-1195753892838600326 x^{8} \\
& +1110278480747979603 x^{7}-721638086761400063 x^{6} \\
& +335025835998692775 x^{5}-111098573305754871 x^{4} \\
& +25789093045603361 x^{3}-3990908419523891 x^{2} \\
& +370559601060925 x-15639435102355 \\
& T(2 / 11,3 / 16)=x^{5}-16175 x^{4}+12295 x^{3}-3502 x^{2}+443 x-21 \\
& T(4 / 13,5 / 16)=x^{9}-369076253174 x^{8}+917724840702 x^{7}-998350365312 x^{6} \\
& +620599596183 x^{5}-241110478731 x^{4}+59951042224 x^{3} \\
& -9316515227 x^{2}+827310070 x-32140845 \\
& T(3 / 7,7 / 16)=x^{3}-37 x^{2}+32 x-7 \\
& T(7 / 16,4 / 9)=-x^{6}-10576186 x^{5}+23312009 x^{4}-20553597 x^{3} \\
& +9060737 x^{2}-1997132 x+176079 \\
& T(1 / 9,2 / 17)=x^{4}-1953 x^{3}+676 x^{2}-78 x+3 \\
& T(1 / 6,3 / 17)=x^{2}-6 x+1 \\
& T(3 / 17,2 / 11)=x^{5}-164752 x^{4}+117596 x^{3}-31475 x^{2}+3744 x-167 \\
& T(3 / 13,4 / 17)=-x^{6}+5654596 x^{5}-6591735 x^{4}+3073650 x^{3}-716598 x^{2} \\
& +83534 x-3895 \\
& T(2 / 7,5 / 17)=-x^{3}-82 x^{2}+48 x-7 \\
& T(5 / 17,3 / 10)=-x^{4}-3986 x^{3}+3547 x^{2}-1052 x+104 \\
& T(1 / 3,6 / 17)=x^{2}-6 x+2
\end{aligned}
$$




$$
\begin{aligned}
& T(6 / 17,5 / 14)=-x^{12}-312068777674512248 x^{11}+1218635449662069926 x^{10} \\
& -2163086830555697775 x^{9}+2303693455082796817 x^{8} \\
& -1635624110167518281 x^{7}+812904818934872080 x^{6} \\
& -288580642413016261 x^{5}+73175550293900447 x^{4}- \\
& -12988595068142207 x^{3}+1536973706418270 x^{2} \\
& -109124254036404 x+3521703559324 \\
& T(2 / 5,7 / 17)=x^{2}+2 x-1 \\
& T(7 / 17,5 / 12)=x^{2}+2 x-1 \\
& T(7 / 15,8 / 17)=-x^{4}+6130 x^{3}-8618 x^{2}+4039 x-631 \\
& T(3 / 11,5 / 18)=x^{5}+303655 x^{4}-334282 x^{3}+137998 x^{2}-25319 x+1742 \\
& T(5 / 18,2 / 7)=-x^{3}-89 x^{2}+50 x-7 \\
& T(5 / 13,7 / 18)=-x^{6}-6049372 x^{5}+11706532 x^{4}-9061607 x^{3}+3507125 x^{2} \\
& -678682 x+52534 \\
& T(7 / 18,2 / 5)=x^{2}-3 x+1 \\
& T(1 / 10,2 / 19)=x^{4}-2710 x^{3}+841 x^{2}-87 x+3 \\
& T(2 / 13,3 / 19)=x^{6}+16300632 x^{5}-12702977 x^{4}+3959686 x^{3}-617135 x^{2} \\
& +48091 x-1499 \\
& T(1 / 5,4 / 19)=x^{2}-5 x+1 \\
& T(4 / 19,3 / 14)=x^{9}-2941000101126 x^{8}+4994011925448 x^{7} \\
& -3710051448922 x^{6}+1574961728536 x^{5}-417866792428 x^{4} \\
& +70955073227 x^{3}-7530205493 x^{2}+456656790 x-12115709 \\
& T(1 / 4,5 / 19)=x^{2}-4 x+1 \\
& T(5 / 19,4 / 15)=x^{4}+3607 x^{3}-2866 x^{2}+759 x-67 \\
& T(5 / 16,6 / 19)=-x^{8}+51931371494 x^{7}-114207671161 x^{6}+107642363378 x^{5} \\
& -56363495447 x^{4}+17707739051 x^{3}-3337942176 x^{2} \\
& +349559613 x-15688671 \\
& T(4 / 11,7 / 19)=-x^{5}-167972 x^{4}+246584 x^{3}-135743 x^{2}+33211 x-3047 \\
& T(7 / 19,3 / 8)=x^{4}+8594 x^{3}-9574 x^{2}+3555 x-440 \\
& T(5 / 12,8 / 19)=x^{6}-13761534 x^{5}+28842552 x^{4} \\
& -24180158 x^{3}+10135687 x^{2}-2124300 x+178089 \\
& T(8 / 19,3 / 7)=x^{3}-107 x^{2}+90 x-19 \\
& T(8 / 17,9 / 19)=-x^{8}+63880292236 x^{7}-211132804023 x^{6}+299066280893 x^{5} \\
& -235345759625 x^{4}+111121028980 x^{3}-31480170773 x^{2} \\
& +4954560070 x-334191985 \\
& T(1 / 7,3 / 20)=x^{3}+231 x^{2}-68 x+5
\end{aligned}
$$




$$
\begin{aligned}
& T(3 / 20,2 / 13)=-x^{6}+28336792 x^{5}-21517541 x^{4}+6535640 x^{3}-992538 x^{2} \\
& +75365 x-2289 \\
& T(1 / 3,7 / 20)=x^{2}-6 x+2 \\
& T(7 / 20,6 / 17)=x^{8}-70615270260 x^{7}+173702478683 x^{6}-183120134018 x^{5} \\
& +107248975216 x^{4}-37687812630 x^{3}+7946199062 x^{2} \\
& -930775452 x+46725407 \\
& T(4 / 9,9 / 20)=x^{3}+224 x^{2}-201 x+45 \\
& T(9 / 20,5 / 11)=-x^{5}-28247 x^{4}+50444 x^{3}-33775 x^{2}+10049 x-1121 \\
& T(1 / 11,2 / 21)=x^{4}-3641 x^{3}+1024 x^{2}-96 x+3 \\
& T(3 / 16,4 / 21)=-x^{8}+136425013870 x^{7}-180508372914 x^{6} \\
& +102358062346 x^{5}-32245743882 x^{4}+6094977959 x^{3} \\
& -691227923 x^{2}+43550791 x-1175959 \\
& T(4 / 17,5 / 21)=x^{6}+1414956 x^{5}-1686559 x^{4}+804089 x^{3}-191673 x^{2} \\
& +22844 x-1089 \\
& T(3 / 8,8 / 21)=x^{2}-3 x+1 \\
& T(8 / 21,5 / 13)=x^{2}-3 x+1 \\
& T(9 / 19,10 / 21)=x^{18}-265066219851470073896475021927 x^{17} \\
& +2140395330694655830972341091874 x^{16} \\
& -8133445821830247750162364615479 x^{15} \\
& +19316795672890032633988244072508 x^{14} \\
& -32113936273710937029720760450948 x^{13} \\
& +39660410718965991151182638887921 x^{12} \\
& -37677096594660667022412296504028 x^{11} \\
& +28123036244133465310172098724688 x^{10} \\
& -16697915529194766473201489538076 x^{9} \\
& +7931442994928916901189904965470 x^{8} \\
& -3013922280150590577654465661841 x^{7} \\
& +911018436460175951387551399941 x^{6} \\
& -216364915651909887212093381346 x^{5} \\
& +39527838685420394912701179067 x^{4} \\
& -5364441433555090728913121916 x^{3} \\
& +509616986326961914742507595 x^{2} \\
& -30258208210601324759757834 x \\
& +845441362748491768882081
\end{aligned}
$$




\section{REFERENCES}

[1] P. Borwein and T. Erdélyi, Polynomials and Polynomial Inequalities, Springer-Verlag, New York, 1995. MR 97e:41001

[2] P. Borwein and T. Erdélyi, The integer Chebyshev problem, Math. Comp. 65 (1996), 661-681. MR 96g:11077

[3] G. V. Chudnovsky, Number theoretic applications of polynomials with rational coefficients defined by extremality conditions, Arithmetic and Geometry, Vol. I (M. Artin and J. Tate, eds.), pp. 61-105, Birkhäuser, Boston, 1983. MR 86c:11052

[4] M. Fekete, Über die Verteilung der Wurzeln bei gewissen algebraischen Gleichungen mit ganzzahligen Koeffizienten, Math. Zeit. 17 (1923), 228-249.

[5] M. Fekete, Über den tranfiniten Durchmesser ebener Punktmengen II, Math. Zeit. 32 (1930), 215-221.

[6] Le Baron O. Ferguson, Approximation by Polynomials with Integral Coefficients, Amer. Math. Soc., Providence, R.I., 1980. MR 81g:41011

[7] V. Flammang, G. Rhin and C.J. Smyth, The integer transfinite diameter of intervals and totally real algebraic integers, J. Théor. Nombres Bordeaux 9 (1997), 137-168. MR 98g:11119

[8] G. M. Goluzin, Geometric Theory of Functions of a Complex Variable, Vol. 26 of Translations of Mathematical Monographs, Amer. Math. Soc., Providence, R.I., 1969. MR 40:308

[9] L. Habsieger and B. Salvy, On integer Chebyshev polynomials, Math. Comp. 218 (1997), 763-770. MR 97f:11053

[10] D. Hilbert, Ein Beitrag zur Theorie des Legendreschen Polynoms, Acta Math. 18 (1894), 155-159.

[11] H. L. Montgomery, Ten Lectures on the Interface Between Analytic Number Theory and Harmonic Analysis, CBMS, Vol. 84, Amer. Math. Soc., Providence, R.I., 1994. MR 96i:11002

[12] Y. Okada, On approximate polynomials with integral coefficients only, Tohoku Math. J. 23 (1924), 26-35.

[13] I. E. Pritsker, Small polynomials with integer coefficients, in press.

[14] T. Ransford, Potential Theory in the Complex Plane, Cambridge University Press, Cambridge, 1995. MR 96e:31001

[15] T. J. Rivlin, Chebyshev Polynomials, John Wiley \& Sons, New York, 1990. MR 92a:41016

[16] R. M. Trigub, Approximation of functions with Diophantine conditions by polynomials with integral coefficients, in "Metric Questions of the Theory of Functions and Mappings", No. 2, Naukova Dumka, Kiev, 1971, pp. 267-333. (Russian) MR 47:683

[17] M. Tsuji, Potential Theory in Modern Function Theory, Chelsea Publ. Co., New York, 1975. MR 54:2990

Department of Mathematics and Statistics, Simon Fraser University, Burnaby, British Columbia, V5A 1S6, Canada

E-mail address: pborwein@cecm.sfu.ca

Department of Mathematics, 138 Cardwell Hall, Kansas State University, ManhatTAN, KANSAS 66506

E-mail address: pinner@math.ksu.edu

Department of Mathematics, 401 Mathematical Sciences, Oklahoma State UniverSity, Stillwater, OKLAhoma 74078

E-mail address: igor@math.okstate.edu 